This manuscript has been submitted for publication in Geophysical Research Letters. We have corrected for the citation in Figure 2. Please note that, the manuscript is currently under review and has yet to be formally accepted for publication. Subsequent versions of this manuscript may have slightly different content. If accepted, the final version of this manuscript will be available via the 'Peer-reviewed Publication DOI' link on the right-hand side of this webpage.

\title{
On the modulation of kinetic energy transfer by internal gravity waves
}

\author{
Adekunle Ajayi, UGA (adeajayi.kunle@gmail.com) \\ Julien Le Sommer, CNRS (julien.Lesommer@univ-grenoble-alpes.fr) \\ Laurent Brodeau, Datlas (lb.ocnnxt@gmail.com) \\ Brian K. Arbic, U. Michigan (arbic@umich.edu) \\ Guillaume Sérazin, CNRS (guillaume.serazin@univ-brest.fr) \\ Aurélie Albert, CNRS (aurelie.albert@univ-grenoble-alpes.fr) \\ Takaya Uchida, CNRS (takaya.uchida@univ-grenoble-alpes.fr) \\ Patrice Klein, Caltech (pklein@caltech.edu)
}




\title{
On the modulation of kinetic energy transfer by internal gravity waves
}

\author{
Adekunle Ajayi ${ }^{1}$, Julien Le Sommer ${ }^{1}$, Laurent Brodeau ${ }^{2,3}$, Brian K. Arbic ${ }^{4}$, \\ Guillaume Sérazin ${ }^{5,6}$, Aurélie Albert ${ }^{1}$, Takaya Uchida ${ }^{1}$ and Patrice Klein ${ }^{7,8}$ \\ ${ }^{1}$ Université Grenoble Alpes/CNRS/IRD/IGE, Grenoble, France \\ ${ }^{2}$ Ocean Next, Grenoble, France \\ ${ }^{3}$ Datlas, Grenoble, France \\ ${ }^{4}$ Department of Earth and Environmental Sciences, University of Michigan, Ann Arbor, MI, USA \\ ${ }^{5}$ Climate Change Research Center, UNSW, Australia \\ ${ }^{6}$ Université de Bretagne Occidentale/CNRS/Ifremer/IRD/LOPS, Plouzané, France \\ ${ }^{7}$ Division of Geological and Planetary Sciences, California Institute of Technology, Pasadena, CA 91106, \\ USA \\ ${ }^{8}$ LMD, Ecole Normale Supérieure/CNRS/IPSL, Paris, France
}

\section{Key Points:}

- Twin submesoscale-permitting simulations of the North Atlantic Ocean were run with and without tidal forcing.

- The tidally-forced run shows enhanced internal gravity wave (IGW) signal during summer while the non-tidally forced run during winter.

- Tidally-forced IGWs (i.e. internal tides) enhance forward cascade of kinetic energy particularly during summertime.

Corresponding author: Takaya Uchida, takaya.uchida@univ-grenoble-alpes.fr 


\begin{abstract}
Understanding how kinetic energy (KE) is exchanged across scales and eventually dissipated remains a key question in physical oceanography. Recent theoretical works suggests that both quasi-balanced submesoscale motions and internal gravity waves (IGWs) could play a role in fluxing KE towards dissipation. How these classes of motions actually provide a route to dissipation in the ocean is still debated. This study investigates the impact of IGWs generated by tidal motions on cross-scale KE exchanges at mid-latitude. Our analysis is based on the output of two realistic submesoscale permitting ocean model simulations of the North Atlantic Ocean, run respectively with and without tidal forcing. These twin experiments permit investigation of how tidally-forced IGWs modify the KE variance, cross-scale exchanges, and associated seasonality. Our results show that, in the presence of externallyforced IGWs, KE transfer towards dissipative scales is enhanced in summertime both at the surface and in the ocean interior.
\end{abstract}

\title{
Plain Language Summary
}

Energetic oceanic currents on the scales of tens of kilometers that emerge as a result of the chaotic nature of the ocean, known as (sub)mesoscale flows, have been of great scientific interest to the oceanographic community. These currents are associated with the time scales of roughly a day, which overlap with the astronomical tidal frequency of the ocean. Due to their similar time scales, it has been argued that the (sub)mesoscale flows and tides would interact with each other. Here, by running a twin numerical simulation of the North Atlantic Ocean, one with tides and the other without, we examine the physical interaction between the two and show that the tides stimulate a loss of energy from the (sub)mesoscale flows particularly during the summer.

\section{Introduction}

The ocean's kinetic energy (KE) is mostly concentrated in motions close to geostrophic balance, with frequencies lower than the Coriolis frequency $(f)$ and spatial scales larger than the first Rossby deformation radius ( $R_{d}$; Vallis, 2017). These balanced motions (BMs) are largely energized through baroclinic instability which extracts energy from large scale stratification. Balanced motions include large-scale motions (>300 km), mesoscale motions $(50-300 \mathrm{~km})$ and submesoscale balanced motions $(<50 \mathrm{~km})($ McWilliams, 2016). Balanced motions are characterized by an inverse cascade of energy (Scott \& Wang, 2005; Scott \& Arbic, 2007; Eden, 2007; Aluie et al., 2017), so they do not provide a route to dissipation by themselves. Therefore, energy has to be transferred from balanced motions to high-frequency 
unbalanced motion for dissipation to occur. To equilibrate the well-known inverse cascade of energy, the ocean requires ageostrophic processes to extract energy from balanced motions. Mechanisms that might trigger a forward transfer of energy from balanced motions down to dissipate scale include (i) bottom boundary-layer turbulence (Wunsch \& Ferrari, 2004; Sen et al., 2008; Arbic et al., 2009), (ii) generation of lee waves by mesoscale eddies interacting with topography (Nikurashin \& Ferrari, 2010; Nikurashin et al., 2013; Trossman et al., 2013, 2016), (iii) generation of internal waves by upper-ocean frontal instabilities (Danioux et al., 2012; Shakespeare \& Taylor, 2014) and (iv) direct cascade of energy by energetic submesoscale motions (Capet et al., 2008; Ferrari \& Wunsch, 2009; Molemaker et al., 2010; McWilliams, 2016). These studies have shown that, at fine-scale, submesoscale motions and internal gravity waves (IGWs) can provide efficient transfer of energy to dissipative scales.

Submesoscale motions are flow structures in the form of density fronts, filaments and topographic wakes at the surface and throughout the interior at scale smaller than $O(100 \mathrm{~km})$ (McWilliams, 2016). IGWs are a particular class of fast propagating unbalanced motions with frequencies equal to or higher than the Coriolis frequency $f$ and spatial scale smaller than $O(100 \mathrm{~km})$. IGWs include wind-induced near-inertial waves with frequency near the Coriolis frequency and internal tides generated by large-scale barotropic tidal flow over topographic features. Near-inertial waves are usually stronger in winter than in summer because they are driven by surface winds (D'Asaro, 1985) while the signature of internal tides (i.e. IGWs with tidal frequencies) is typically stronger in summer time (Gerkema et al., 2004). Recent works have highlighted that these two classes of motions-submesoscale motions and IGWs-are out of seasonally phase (Rocha et al., 2016). Submesoscale motions tend to be stronger than IGWs in wintertime. The emergence of submesoscales is due to winter favored mechanisms such as mixed layer instability and wind-induced frontal instability (e.g. Qiu et al., 2014; Sasaki et al., 2014; Callies, Flierl, et al., 2015; Brannigan et al., 2015; McWilliams, 2016; Uchida et al., 2019; Khatri et al., 2021). KE associated with IGWs shows stronger amplitude in summertime. This is due to the intensification of vertical normal modes and shallow mixed layer (Callies, Ferrari, et al., 2015; Rocha et al., 2016).

A forward cascade is seen in spectral KE fluxes computed from gridded satellite altimeter products (Scott \& Wang, 2005) at scales smaller than $O(100 \mathrm{~km})$. Later work (Arbic et al., 2013) argued that while this forward cascade could be physically based, and related to energy transfer between mesoscale eddies and IGWs, it could also be due to the spatial and temporal smoothing inherent in the construction of gridded altimeter products. Due to high-frequency winds and tidal motions, energy extraction via IGWs seems to be a highly probable mechanism of KE sinks for balanced motions (J. Thomas \& Daniel, 2021). There 
have so far been two well documented energy transfer mechanisms from balanced motions to IGWs away from topography, namely the stimulated generation of a forward cascade of KE by near-inertial waves from balanced flows (Gertz \& Straub, 2009; Rocha et al., 2018; Barkan et al., 2021), and spontaneous generation of near-inertial waves from balanced flows (Nagai et al., 2015; Shakespeare \& Hogg, 2017). In stimulated generation, near-inertial waves are first introduced by external forcing (e.g. wind) and then grow by extracting energy from the balanced flow (Gertz \& Straub, 2009; Barkan et al., 2017; L. N. Thomas, 2017) while spontaneous generation is the emission of waves by unbalanced, large Rossby number flow at density fronts without external forcing. These waves then radiate vertically downwards into the interior and amplify by extracting energy from deep balanced flow (Shakespeare \& Hogg, 2017). Spontaneous generation is localized at sharp submesoscale fronts and is inefficient at small Rossby numbers (Danioux et al., 2012; Nagai et al., 2015; Shakespeare \& Hogg, 2017). On the other hand, stimulated generation is efficient at small Rossby numbers provided that the waves are forced externally.

As listed above, several studies have focused on the impact of wind-generated nearinertial waves on energy dissipation (e.g. Barkan et al., 2017; J. Thomas \& Arun, 2020). In contrast, little is known as to the role of internal tides on KE exchanges (cf. Barkan et al., 2021). We know that internal tides contribute to the building up of IGW continuum (Garrett \& Munk, 1975) and that they eventually contribute to diapycnal mixing in the ocean interior (Arbic et al., 2018). Whether internal tides could play a significant role in the down-scale transfer of mesoscale eddy $\mathrm{KE}$ is yet to be fully explored. In this context, we investigate the role of internal tides on cross-scale $\mathrm{KE}$ exchanges in a regime with active submesoscale motions by using a twin submesoscale resolving numerical simulations (with/without tides) of the North Atlantic Ocean with a horizontal resolution of $1 / 60^{\circ}$. This permits us to investigate how IGWs affect KE exchanges in the presence of active submesoscales motions.

This paper is organized as follows: in the next section, we describe the numerical simulations. Section 3 presents the seasonality of BMs and IGWs characterized by the KE frequency-wavenumber spectral density. The contribution of BMs and IGWs to the KE transfer is presented in section 4 . We discuss the impacts of this observed seasonality on the KE spectral flux in section 5, and we identify two different mechanisms that support a direct cascade of energy in a dynamical regime with/without tidal motions.

\section{North Atlantic Ocean Simulation}

In this study, we use numerical outputs from a NEMO-based submesoscale eddy-permitting simulations of the North Atlantic with a horizontal resolution of 1/60 (eNATL60; Brodeau et al., 2020). eNATL60 is a spatially-extended version of NATL60 (Ajayi et al., 2020, 
2021). The simulation spans the North Atlantic Ocean from about $6^{\circ} \mathrm{N}$ up to the polar circle and has a horizontal grid spacing ranging from $1.6 \mathrm{~km}$ at $6^{\circ} \mathrm{N}$ to $0.9 \mathrm{~km}$ at $65^{\circ} \mathrm{N}$. The model has 300 vertical levels with a resolution of $1 \mathrm{~m}$ at the top-most layers to better resolve a realistic surface boundary layer. In practice, the model effective resolution is about $10-15 \mathrm{~km}$ in wavelength, the same as the resolution of the anticipated Surface Water and Ocean Topography (SWOT) altimetry mission (Fu \& Ubelmann, 2014). The initial and open boundary conditions are based on GLORYS2v3 ocean reanalysis with a relaxation zone at the northern boundary for sea-ice concentration and thickness. The atmospheric forcing is based on ERA-Interim (ECMWF; Dee et al., 2011), the grid and bathymetry follow (Ducousso et al., 2017). A third-order upwind advection scheme is used for both momentum and tracers in the model simulation to implicitly adapt lateral viscosity and diffusivity to flow properties. The model is spun up for 18 months, and a one-year simulation output from July 2009 to June 2010 is used in this study. eNATL60 has two identical runs (i) eNATL60 with tidal forcing herein referred to as eN60-WT and (ii) eNATL60 with no tidal forcing eN60-NT. The two simulations have perfectly the same configuration except for the inclusion of tidal motions in eN60-WT. In the rest of this article, we use eNATL60 to refer to the two simulations while individual runs are addressed as eN60-WT (with tides) or eN60-NT (no tides). The inclusion of tidal forcing in eN60-WT run provides conversion of tidal energy into the internal wave field through, both, flow-topography interactions and wave-balanced motions interactions (Arbic et al., 2008, 2018).

To investigate cross-scale energy exchanges between different scales of motions, we estimate KE spectral density in frequency-wavenumber space as a proxy to understand the energetic nature of balanced/unbalanced motions in regimes with/without tidal motions. Also, we estimate the rate at which nonlinear mechanisms exchange energy across temporal and spatial scales in the two scenarios. In what follows, our analysis of KE density and transfer is based on the hourly output of horizontal total velocity field and are computed using the following equation;

$$
\begin{gathered}
\frac{\partial \widehat{K E}}{\partial t}=T_{K E}+\widehat{\mathbf{u}}^{*} \cdot O T \\
\widehat{K E}=\frac{1}{2} \widehat{\mathbf{u}}^{*} \cdot \widehat{\mathbf{u}} \\
T_{K E}=-\widehat{\mathbf{u}}^{*} \cdot[\widehat{\mathbf{u}} \widehat{\cdot \nabla \mathbf{u}}]
\end{gathered}
$$

Equations (1)-(3) are derived from the Fourier transform of momentum equation multiplied by horizontal velocity field (Scott \& Wang, 2005; Capet et al., 2008; Müller et al., 2015). In the momentum equation (Equation 1), $K E$ and $T_{K E}$ represents the KE density and transfer respectively while $O T$ stands "Other Terms". [] refers to Fourier transform and $*$ represents the complex conjugate. Before performing spectral analysis the $2 \mathrm{D}$ time series were de- 
trended and windowed in space and time consistent with standard procedures previously used in Rocha et al. (2016), Müller et al. (2015) and Torres et al. (2018).

The eNATL60 simulation resolves well to a reasonable extent, mesoscale motions, submesoscale motions, and IGWs (see Figure 1 in SI). The comparison of eNATL60 sea surface height (SSH) spectral density with SARAL AltiKa (Figure 1a) shows that the predicted SSH variance by the model compares well with the satellite observation for scales $>100 \mathrm{~km}$. There are differences at scales $<100 \mathrm{~km}$ that are due to the satellite instrument noise level. There seems to be quite a robust agreement between the two runs of eNATL60 simulations in wintertime. However, of particular interest is the difference between the runs in summertime where variance at fine-scales is of higher magnitude in eN60-WT than to eN60-NT.

A similar analysis of the KE spectral density in the same region (Figure 1b), shows that the variance associated with fine-scale motions smaller than $100 \mathrm{~km}$ is higher in the eN60WT compared to eN60-NT. So what are the mechanism/dynamics at fine-scales in eN60WT that could be responsible for this higher variance? A possible answer to this is that the inclusion of tidal motion in eN60-WT simulation is responsible for enhanced wave activity, and this is why we see higher variance at fine scales in the SSH and KE spectra density plot. To qualitatively investigate this, we separate the flow into its rotational and divergent part, which represents the balanced and the unbalanced wave motions, respectively. Figure 1c presents the spectral density for these two components. The spectra of the rotational part for the two runs are almost indistinguishable, indicating that both simulations have nearly equal energy levels in geostrophically balanced motions. However, the divergence spectra of the KE is very different between the two simulations. This difference is obvious at scales less than $500 \mathrm{~km}$, and indeed, the divergent motions are more energetic in eN60-WT by a factor of 2 with two interesting peaks. We can conclude that the higher variance in eN60WT at fine-scales compared to eN60-NT is primarily due to stronger divergent motions in eN60-WT, which is caused by the inclusion of tidal forcing in this simulation.

\section{Seasonality of BMs and IGWs}

This section presents the different classes of motions and their seasonality based on frequency-wavenumber $(\omega-K)$ spectral density. This diagnostic will help us better understand how the difference in wave activity between the two simulations affects oceanic motions' spectral signature across different temporal and spatial scales. Following Torres et al. (2018), we begin by presenting a schematic (Figure 2a) showing the different observable dynamical regimes in the ocean as a function of their temporal and spatial scale. These classes of motions starting with low-frequency, low-wavenumber motions to high-frequency, high-wavenumber motions are Rossby waves (RW), mesoscale balanced motions (MBM), 
submesoscale balanced motions (SBM), unbalanced submesoscale motions (USM) and internal gravity waves (IGW). Due to the computational cost of this diagnostic tool, we perform the $\omega-K$ spectral analysis in a $5^{\circ} \times 5^{\circ}\left(-40^{\circ}\right.$ to $-35^{\circ}, 40^{\circ}$ to $\left.45^{\circ}\right)$ box located inside the previous large box (see Figure 1 of SI).

We also show, in Figure 2, the winter and summer averages of surface KE $\omega-K$ spectra for the two runs. The classes of motions described previously in Figure 2a are identifiable in the figure. The winter-summer contrast shows a strong seasonality in the spectral density of SBMs and IGWs. In wintertime, for eN60-WT, energy is mostly concentrated in BMs, near-inertial waves, and the dispersion curve of IGWs (Figure 2b), while in summertime, energy is mostly concentrated in the MBMs, near-inertial waves, and internal tides (Figure 2d). In particular, the variance associated with SBMs is stronger in winter, while that of IGWs is stronger in summer. Our understanding is that IGWs are stronger in summer due to shallow mixed layer depth and vertical normal mode intensification. Simultaneously, SBMs are stronger in winter because they are driven by winter-favored mechanisms such as frontogenesis, wind-induced frontal instabilities, and mixed layer instability, among other processes (Qiu et al., 2014; Sasaki et al., 2014; Callies, Flierl, et al., 2015; Brannigan et al., 2015; McWilliams, 2016; Uchida et al., 2019; Khatri et al., 2021). This out of phase seasonality of SBMs and IGWs is consistent with the findings of Rocha et al. (2016) and Torres et al. (2018).

Similarly, eN60-NT resolves the same classes of motion as eN60-WT except that internal tides are absent and supertidal IGWs are less energetic. In wintertime, energy is mostly concentrated in BMs, near-inertial waves, and along the dispersion curve of IGWs (Figure 2a). This is consistent with the winter dynamics in eN60-WT. In summertime, energy is concentrated in MBMs and near-inertial waves (Figure 2c). Unlike eN60-WT, the seasonality observed in eN60-NT is associated with stronger SBMs and IGWs in winter. The seasonality of IGWs is reversed in eN60-NT when compared to eN60-WT. How can this be? We know that the classical paradigm for the generation of the supertidal IGW continuum is that winds produce near-inertial waves, barotropic tidal flow over topographic features creates internal tides, and the energy along the IGW dispersion curve is due to nonlinear interactions. Both simulations are forced with realistic high-frequency winds with 3-hourly outputs (Brodeau et al., 2020). These winds are stronger in winter, hence there is a well-resolved near-inertial wave and IGWs dispersion curve in winter. The dynamics in summertime are different between the two runs. For the eN60-WT simulation, internal tides generated by tidal motions are amplified by a shallow mixed layer in summertime, and nonlinearity produces energy in the IGW dispersion curve. Thus for eN60-NT, the mechanism for generating waves in summertime is relatively weak; no tidal forcing and weaker winds, 
hence relatively weak wave motions in summer. The difference in the spectral energy density of different dynamical regimes between the two simulations extends below the surface (see Figure 3 of SI).

To obtain frequency spectra, we integrate the $\omega-K$ spectra over all wavenumbers for the two runs (Figure 3). In summertime, the variance at high frequencies $\left(\mathrm{M}_{2}\right.$ and supertidal frequencies) is higher in eN60-WT compared to eN60-NT. We believe that this is likely due to the amplification of IGWs by tidal motions. eN60-WT spectra approximately follow the estimated Garrett-Munk spectra (Garrett \& Munk, 1975; Cairns \& Williams, 1976; Müller et al., 2015) in summertime. Visible in eN60-WT spectra are the peaks at the inertia frequency $f$ and the $M_{2}$ tidal frequency. In contrast, only the near-inertial peak is visible in eN60-NT. To a large extent, we now understand the dynamics responsible for the differences in KE density that we see in the two simulations. In the following sections, we shall discuss how the enhanced IGWs arising from tidal forcing affect the redistribution of energy in different dynamical regimes.

\section{Modulation of KE forward flux by IGWs}

In this section, we will discuss the impact of resolving internal tides on the magnitude and direction of $\mathrm{KE}$ cascade at fine-scales in the wavenumber and frequency-wavenumber domain by comparing the twin eNATL60 runs.

\subsection{Wavenumber KE flux}

We start by examining the KE flux in the wavenumber domain. We do this by estimating the net energy (spectral flux) passing through individual wavenumbers in spectral space. The spectral flux is obtained by integrating the energy transfer (Equation 3) from a particular wavenumber $K$ to $K_{0}$ (the wavenumber corresponding to the box size).

We present in Figure 4a,d, the winter and summer averages of KE spectral flux for both simulations. In wintertime and in the two simulations (Figure 4a), the flux is nearly identical across all wavenumbers. The forward cascade starts at around $25 \mathrm{~km}$ and extends down to a kilometric scale. In summertime (Figure 4d), the magnitude of the forward cascade at high wavenumbers differs significantly between the two runs. The inclusion of tidal forcing in eN60-WT yields a forward flux at high wavenumbers that is a factor of 4 higher than the forward high-wavenumber flux in eN60-NT. This difference in cascade highlights how internal tides enhance the forward cascade of $\mathrm{KE}$ at high wavenumbers.

Thus far, the KE cascade has been estimated only at the surface. Considering we have three-dimensional information of the ocean, as opposed to satellite observations, it 
is of great interest to understand the nature of the KE cascade in the ocean's interior. In Figure 4b,c,e,f, we present the spectral flux computed at 32 different vertical levels in the upper $1000 \mathrm{~m}$ of the water column. In winter and summer, at lower wavenumbers, the average $\mathrm{KE}$ flux in the two simulations is characterized by a net inverse cascade that extends down to around $700 \mathrm{~m}$ in the interior. In wintertime, the forward cascade at higher wavenumbers in eN60-WT (Figure 4c) is strong both at the surface and in the interior. In contrast, in eN60-NT (Figure 4b), the forward cascade is confined mostly to the surface. In summertime, the forward cascade in eN60-WT (Figure 4f) span the upper ocean but with a gradual decrease in magnitude farther down the water column. In contrast, in eN60-NT (Figure 4e), the forward cascade is nearly absent throughout the upper ocean. A stronger forward cascade (in summertime for eN60-WT) in the interior is an indication that internal tides transfer substantial amounts of KE to dissipative scales throughout the upper ocean water column.

\subsection{Frequency-wavenumber KE transfer}

We have demonstrated that internal tides enhance the supertidal IGW continuum and, in particular, enhance the forward cascade of energy in summertime. To better explain how internal tides modify cross-scale energy exchanges among the different classes of motions, we present in Figure 5 the winter and summer averages of KE spectral transfer in frequencywavenumber space. In the $\omega-K$ spectra, negative values of spectra transfer imply that non-linearity extracts energy from these regions to feed other regions with positive values. In other words, sinks of energy are characterized by positive values, while sources of energy have negative values.

We start by discussing the spectral transfer in wintertime (cf. Equation 3; Figures 5a,c). In eN60-NT, the spectral transfer's positive values (blue) show that balanced motions serve as a source of energy for other motions, namely energy is being extracted from the balanced motions. In contrast, near-inertial motions and motions with scales less than $10 \mathrm{~km}$ are the major sinks of KE (red). The rate of non-linear exchanges in eN60-WT is similar to eN60-NT except for the mild intensification of energy gained by IGWs in eN60-WT. To summarize, submesoscale motions and internal gravity waves are sinks of KE in wintertime, with the former playing the major role.

The summer spectra (Figure 5b,d) differ significantly between the two runs as expected. In eN60-NT, BMs represent the major source of energy for other motions, while energy is gained mostly by near-inertial motions. The transfer at high frequencies and wavenumbers is very small. We can interpret this to signify that high-frequency motions and submesoscale motions are less energetic in eN60-NT in summertime. This result is consistent with results 
in the KE spectra density. However in eN60-WT, the situation is different. The major energy source for other motions is the MBMs and the semi-diurnal tides (blue). Nearinertial waves, SBMs and the supertidal IGW continuum spectrum are seen in Figure 5 to be gaining energy. The extraction of semi-diurnal tidal energy, and gain of energy in the IGW continuum, due to nonlinear interactions was also seen in (Müller et al., 2015) but is more clear here, perhaps because of the finer vertical and horizontal grid spacing in eN60-WT.

In summary, there are two mechanisms of energy extraction in summertime: (i) without tidal forcing, near-inertial waves gain energy from BMs, and (ii) with tidal forcing, nearinertial waves and the supertidal IGW continuum gain energy from internal tides and MBMs. In summary, the forward cascade in eN60-WT, is associated with the transfer of energy by nonlinearity from balanced motions and internal tides to near-inertial waves and the supertidal IGW continuum. This transfer is possible due to the intensification of IGWs in the presence of tidal motions. The lack of tidal motions in eN60-NT (compared to eN60WT) shows how effective internal tides are in providing a route to energy dissipation in summertime, both at the surface and in the interior. This result strongly emphasizes the need to include tidal forcing in ocean model simulations to accurately predict cross-scale energy exchanges at fine-scales.

\section{Summary and conclusion}

The role of internal tides in the seasonality of KE density and spectral KE transfer was investigated in this study. Our analysis was based on the output of a realistic NEMO simulation of the North Atlantic Ocean with a horizontal resolution of $1 / 60^{\circ}$. We used two outputs of this numerical experiment; one with, and one without, tidal forcing. These twin experiments permit investigation of how IGWs generated by tidal forcing modify KE variance, cross-scale exchanges, and associated seasonality. In the simulation without tides, IGWs are stronger in winter, whereas, in simulation with tides, they are stronger in summer. The latter condition is consistent with the findings of Rocha et al. (2016) and Torres et al. (2018).

Our results also show that resolving internal tides in the presence of energetic submesoscale motions has a strong impact on KE transfer in summertime (cf. Barkan et al., 2021, their Figure 3d,f). The magnitude of the estimated forward cascade at high wavenumbers (both at the surface and at depth) in the simulation with tidal forcing is a factor 4 higher than in the simulation without tidal forcing (Figure 4). Overall, we identified that two mechanisms supporting the KE forward cascade; (i) forward cascade due to energetic submesoscale motions in wintertime and (ii) forward cascade due to IGWs (enhanced by tidal 
forcing) in summertime (Figure 5). Our results underscore that at fine-scales, internal tides can provide an effective route to KE dissipation.

In light of the SWOT altimeter mission (Morrow et al., 2019; Torres et al., 2019), the difference between the runs with tidal forcing and without (e.g. Figures 1 and 3) highlight the importance of tidal forcing in emulating the upcoming altimeter observations (Arbic et al., 2018; Barkan et al., 2021; Yu et al., 2021).

\section{Open research}

The twin eNATL60 data are permanently stored at the CINES supercomputing center in Montpellier, France. The data are available via OPeNDAP (doi:10.5281/zenodo.5910038).

\section{Acknowledgments}

AAj was partly funded by the Université Grenoble Alpes AGIR research grant. JLS, $\mathrm{LB}$ and $\mathrm{AAl}$ acknowledge the PRACE $16^{\text {th }}$ call project ReSuMPTiOn (Revealing SubMesoscale Processes and Turbulence in the Ocean, P.I.: LB) for awarding access to the MareNostrum supercomputer at the Barcelona Supercomputing Center. JLS acknowledges support from Centre National d'Etudes Spatiales (MIDAS project) and Agence Nationale pour la Recherche (ANR-17-CE01-0009-01). BKA acknowledges support from NASA grants NNX16AH79G, NNX17AH55G, and 80NSSC20K1135. BKA began collaborations with the Grenoble group while on sabbatical in France, and he thanks many French colleagues, especially Thierry Penduff, Rosemary Morrow, and Nadia Ayoub, for their help in procuring this sabbatical year. PK is supported by the NASA NPP Senior Fellowship and the SWOT and SMode projects. TU acknowledges support from the French 'Make Our Planet Great Again' (MOPGA) initiative managed by ANR under the Programme d'Investissement d'Avenir, with the reference ANR-18-MPGA-0002. 


\section{References}

Ajayi, A., Le Sommer, J., Chassignet, E., Molines, J., Xu, X., Albert, A., \& Cosme, E. (2020). Spatial and Temporal Variability of the North Atlantic Eddy Field From Two Kilometric-Resolution Ocean Models. J. Geophys. Res. Ocean., 125(5). doi: 10.1029/2019jc015827

Ajayi, A., Le Sommer, J., Chassignet, E., Molines, J., Xu, X., Albert, A., \& Dewar, W. (2021). Diagnosing crossscale kinetic energy exchanges from two submesoscale permitting ocean models. JAMES. doi: 10.1029/2019MS001923

Aluie, H., Hecht, M., \& Vallis, G. K. (2017). Mapping the Energy Cascade in the North Atlantic Ocean: The Coarse-graining Approach. J. Phys. Oceanogr., 225-244. doi: 10.1175/JPO-D-17-0100.1

Arbic, B. K., Alford, M., Ansong, J., Buijsman, M., Ciotti, R., Farrar, J., ... Zhao, Z. (2018). A primer on global internal tide and internal gravity wave continuum modeling in HYCOM and MITgcm. In E. Chassignet, A. Pascual, J. Tintoré, \& J. Verron (Eds.), New frontiers in operational oceanography (pp. 307-392). GODAE Ocean View. doi: 10.17125/gov2018.ch13

Arbic, B. K., Mitrovica, J. X., MacAyeal, D. R., \& Milne, G. A. (2008). On the factors behind large Labrador Sea tidesduring the last glacial cycle and the potential implications for Heinrich events. Paleoceanography, 23.

Arbic, B. K., Polzin, K. L., Scott, R. B., Richman, J. G., \& Shriver, J. F. (2013). On eddy viscosity, energy cascades, and the horizontal resolution of gridded satellite altimeter products. J. Phys. Oceanogr., 43, 283-300. doi: 10.1175/JPO-D-11-0240.1

Arbic, B. K., Shriver, J. F., Hogan, P. J., Hurlburt, H. E., McClean, J. L., Metzger, E. J., ... Wallcraft, A. J. (2009). Estimates of Bottom Flows and Bottom Boundary Layer Dissipation of the Oceanic General Circulation from Global High-Resolution Models. J. Geophys. Res., 114, C02024. doi: 10.1029/2008JC005072

Barkan, R., Srinivasan, K., Yang, L., McWilliams, J. C., Gula, J., \& Vic, C. (2021). Oceanic mesoscale eddy depletion catalyzed by internal waves. Geophysical Research Letters, 48(18), e2021GL094376. doi: 10.1029/2021GL094376

Barkan, R., Winters, K. B., \& McWilliams, J. C. (2017). Stimulated Imbalance and the Enhancement of Eddy Kinetic Energy Dissipation by Internal Waves. J. Phys. Oceanogr., $47(1), 181-198$.

Brannigan, L., Marshall, D. P., Naveira-Garabato, A., \& George Nurser, A. J. (2015). The seasonal cycle of submesoscale flows. Ocean Modelling, 92, 69-84. doi: 10.1016/ j.ocemod.2015.05.002

Brodeau, L., Albert, A., \& Le Sommer, J. (2020). NEMO-eNATL60 description and assessment repository. doi: 10.5281/zenodo.4032732 
Cairns, J., \& Williams, G. (1976). Internal wave observations from a midwater float. JAMES, 81 (12), 1943 1950. doi: 10.1029/JC081i012p01943

Callies, J., Ferrari, R., Klymak, J. M., \& Gula, J. (2015). Seasonality in submesoscale turbulence. Nature Communication, 6, 6862 .

Callies, J., Flierl, G., Ferrari, R., \& Fox-Kemper, B. (2015). The role of mixed-layer instabilities in submesoscale turbulence. J. Fluid Mech., 788, 5 - 41.

Capet, X., Klein, P., Hua, B. L., Lapeyre, G., \& McWilliams, J. C. (2008). Surface kinetic energy transfer in surface quasi-geostrophic flows. J. Fluid Mech., 604, 165-174.

Danioux, E., Vanneste, J., Klein, P., \& Sasaki, H. (2012). Spontaneous inertia-gravitywave generation by surface-intensified turbulence. Journal of Fluid Mechanics, 699, 153173.

D'Asaro, E. A. (1985). The Energy Flux from the Wind to Near-Inertial Motions in the Surface Mixed Layer. J. Phys. Oceanogr., 15, 10431059.

Dee, D. P., Uppala, S. M., Simmons, A., Berrisford, P., Poli, P., Kobayashi, S., ... others (2011). The era-interim reanalysis: Configuration and performance of the data assimilation system. Quarterly Journal of the royal meteorological society, 137(656), 553-597. doi: 10.1002/qj.828

Ducousso, N., Le Sommer, J., Molines, J. M., \& Bell, M. (2017). Impact of the symmetric instability of the computational kind at mesoscale- and submesoscale-permitting resolutions. Ocean Modelling, 120(18 26).

Eden, C. (2007). Eddy length scales in the North Atlantic Ocean. Journal of Geophysical Research, 112(C6), C06004.

Ferrari, R., \& Wunsch, C. (2009). Ocean Circulation Kinetic Energy: Reservoirs, Sources, and Sinks. Annu. Rev. Fluid Mech., 41(1), 253-282.

Fu, L. L., \& Ubelmann, C. (2014). On the transition from profile altimeter to swath altimeter for observing global ocean surface topography. J. Atmos. Oceanic Technol, 31,560568 .

Garrett, C., \& Munk, W. (1975). Space-time scales of internal waves: A progress report. JAMES, 80(3), 291 297. doi: 10.1029/JC080i003p00291

Gerkema, T., Lam, F.-P. A., \& Maas, L. R. (2004). Internal tides in the Bay of Biscay: conversion rates and seasonal effects. Deep Sea Research Part II: Topical Studies in Oceanography, 51(25-26), 2995-3008. doi: 10.1016/j.dsr2.2004.09.012

Gertz, A., \& Straub, D. N. (2009). Near-Inertial Oscillations and the Damping of Midlatitude Gyres: A Modeling Study. J. Phys. Oceanogr., 39(9), 2338-2350. doi: 10.1175/2009jpo4058.1

Khatri, H., Griffies, S. M., Uchida, T., Wang, H., \& Menemenlis, D. (2021). Role of mixedlayer instabilities in the seasonal evolution of eddy kinetic energy spectra in a global 
submesoscale permitting simulation. Geophysical Research Letters, e2021GL094777. doi: 10.1029/2021GL094777

McWilliams, J. C. (2016). Submesoscale currents in the ocean. Proc. R. Soc., A 472, 20160117.

Molemaker, M. J., McWilliams, J. C., \& Capet, X. (2010). Balanced and unbalanced routes to dissipation in an equilibrated eady flow. Journal of Fluid Mechanics, 654, 35-63. doi: $10.1017 /$ S0022112009993272

Morrow, R., Fu, L.-L., Ardhuin, F., Benkiran, M., Chapron, B., Cosme, E., ... Zaron, E. D. (2019). Global Observations of Fine-Scale Ocean Surface Topography With the Surface Water and Ocean Topography (SWOT) Mission. Frontiers in Marine Science, 6. doi: $10.3389 /$ fmars.2019.00232

Müller, M., Arbic, B. K., Richman, J. G., Shriver, J. F., Kunze, E. L., Scott, R. B., ... Zamudio, L. (2015). Toward an internal gravity wave spectrum in global ocean models. Geophys. Res. Lett., 42, 34743481.

Nagai, T., Tandon, A., Kunze, E., \& Mahadevan, A. (2015). Spontaneous Generation of Near-Inertial Waves by the Kuroshio Front. J. Phys. Oceanogr., 45 (9), 2381-2406. doi: $10.2139 /$ ssrn.2906136

Nikurashin, M., \& Ferrari, R. (2010). Radiation and Dissipation of Internal Waves Generated by Geostrophic Motions Impinging on Small-Scale Topography: Theory. J. Phys. Oceanogr., 40(9), 2025-2042. doi: 10.1175/2010JPO4315.1

Nikurashin, M., Vallis, G. K., \& Adcroft, A. (2013). Routes to energy dissipation for geostrophic flows in the Southern Ocean. Nat. Geosci., 6(1), 48-51. doi: 10.1038/ ngeo1657

Qiu, B., Chen, S., Klein, P., Sasaki, H., \& Sasai, Y. (2014). Seasonal Mesoscale and Submesoscale Eddy Variability along the North Pacific Subtropical Countercurrent. Journal of Physical Oceanography, 44 (12), 3079 - 3098.

Rocha, C. B., Gille, S. T., Chereskin, T. K., \& M., D. (2016). Seasonality of submesoscale dynamics in the Kuroshio Extension. Geophysical Research Letters, 43, 11304 - 11311.

Rocha, C. B., Wagner, G. L., \& Young, W. R. (2018). Stimulated generation: Extraction of energy from balanced flow by near-inertial waves. J. Fluid Mech., 847, 417-451.

Sasaki, H., Klein, P., Qiu, B., \& Sasai, Y. (2014). Impact of oceanic scale- interactions on the seasonal modulation of ocean dynamics by the atmosphere. Nature Communication, 5,5636 .

Scott, R. B., \& Arbic, B. K. (2007). Spectral Energy Fluxes in Geostrophic Turbulence: Implications for Ocean Energetics. J. Phys. Oceanogr., 37(3), 673-688. Retrieved from http://journals.ametsoc.org/doi/abs/10.1175/JP03027.1 doi: 10.1175/ JPO3027.1 
Scott, R. B., \& Wang, F. (2005). Direct Evidence of an Oceanic Inverse Kinetic Energy Cascade from Satellite Altimetry. J. Phys. Oceanogr., 35, 1650-1666. Retrieved from http://journals.ametsoc.org/doi/abs/10.1175/JP02771.1 doi: 10.1175/ JPO2771.1

Sen, A., Scott, R. B., \& Arbic, B. K. (2008). Global Energy Dissipation Rate of DeepOcean Low-Frequency Flows by Quadratic Bottom Boundary Layer Drag: Computations from Current Meter Data. Geophys. Res. Lett., 35, L09606. doi: 10.1029/ 2008GL033407

Shakespeare, C. J., \& Hogg, A. M. (2017). Spontaneous Surface Generation and Interior Amplification of Internal Waves in a Regional-Scale Ocean Model. J. Phys. Oceanogr., $47(4), 811-826$.

Shakespeare, C. J., \& Taylor, J. R. (2014). The spontaneous generation of inertia-gravity waves during frontogenesis forced by large strain: Theory. J. Fluid Mech., 757, 817 853.

Thomas, J., \& Arun, S. (2020). Near-inertial waves and geostrophic turbulence. Physical Review Fluids, 5(1), 014801. doi: 10.1103/PhysRevFluids.5.014801

Thomas, J., \& Daniel, D. (2021). Forward flux and enhanced dissipation of geostrophic balanced energy. Journal of Fluid Mechanics, 911. doi: 10.1017/jfm.2020.1026

Thomas, L. N. (2017). On the modifications of near-inertial waves at fronts: implications for energy transfer across scales. Ocean Dyn., 67(10), 1335-1350.

Torres, H. S., Klein, P., Menemenlis, D., Qiu, B., Su, Z., Wang, J., .. Fu, L. L. (2018). Partitioning Ocean Motions Into Balanced Motions and Internal Gravity Waves: A Modeling Study in Anticipation of Future Space Missions. J. Geophys. Res. Ocean., 123, 8084-8105. doi: 10.1029/2018JC014438

Torres, H. S., Klein, P., Siegelman, L., Qiu, B., Chen, S., Ubelmann, C., ... Fu, L.-L. (2019). Diagnosing ocean-wave-turbulence interactions from space. Geophysical Research Letters, 46 (15), 8933-8942. doi: 10.1029/2019GL083675

Trossman, D. S., Arbic, B. K., Garner, S. T., Goff, J. A., Jayne, S. R., Metzger, E. J., \& Wallcraft, A. J. (2013). Impact of Parameterized Lee Wave Drag on the Energy Budget of an Eddying Global Ocean Model. Ocean Modelling, 72, 119-142. doi: 10.1016/j.ocemod.2013.08.006

Trossman, D. S., Arbic, B. K., Richman, J. G., Garner, S. T., Jayne, S. R., \& Wallcraft, A. J. (2016). Impact of Topographic Internal Lee Wave Drag on an Eddying Global Ocean Model. Ocean Modelling, 97, 109-128. doi: 10.1016/j.ocemod.2015.10.013

Uchida, T., Balwada, D., Abernathey, R., McKinley, G., Smith, S., \& Levy, M. (2019). The contribution of submesoscale over mesoscale eddy iron transport in the open southern ocean. Journal of Advances in Modeling Earth Systems, 11(12), 3934-3958. doi: 
10.1029/2019MS001805

Vallis, G. K. (2017). Atmospheric and oceanic fluid dynamics (2nd ed.). Cambridge University Press.

Wunsch, C., \& Ferrari, R. (2004). Vertical mixing, energy, and the general circulation of the oceans. Annu. Rev. Fluid Mech., 36(4), 281 - 314.

Yu, X., Ponte, A. L., Lahaye, N., Caspar-Cohen, Z., \& Menemenlis, D. (2021). Geostrophy assessment and momentum balance of the global oceans in a tide-and eddy-resolving model. Journal of Geophysical Research: Oceans, 126(10), e2021JC017422. doi: 10.1029/2021JC017422 

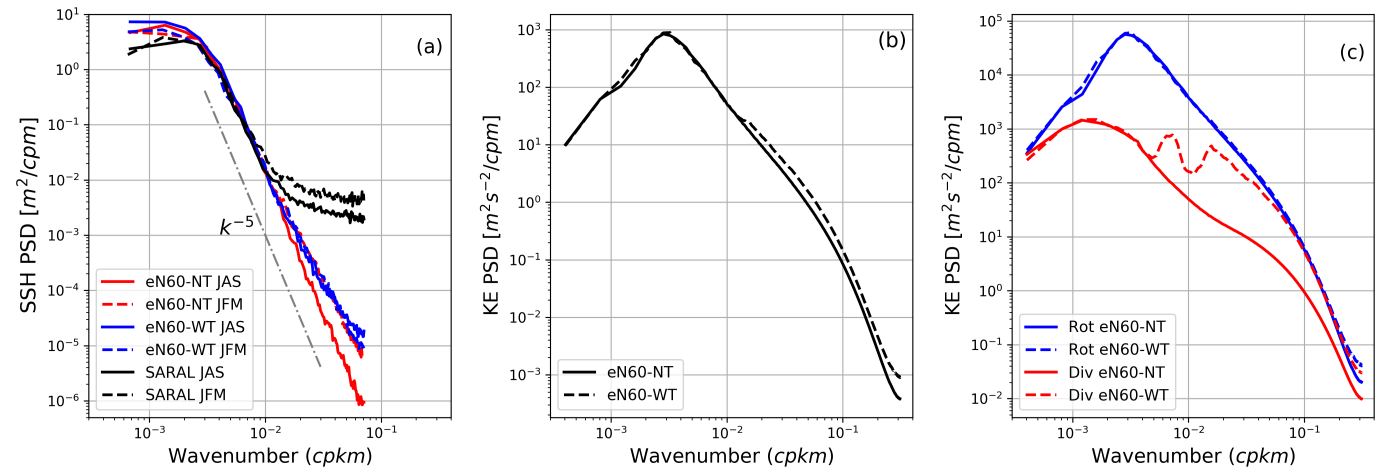

Figure 1. (a) Comparison of SSH wavenumber spectra between eNATL60 and SARAL AltiKa satellite. (b) One year average of surface KE wavenumber spectral density computed from hourly outputs of eN60-NT (no tides) and eN60-WT (with tides). (c) Helmholtz decomposition of KE into rotational $(\zeta)$ and divergent $(\delta)$ spectral components. Thick curves represent the simulation without tides (eN60-NT) and dashed curves represent the simulation with tides (eN60-WT). 

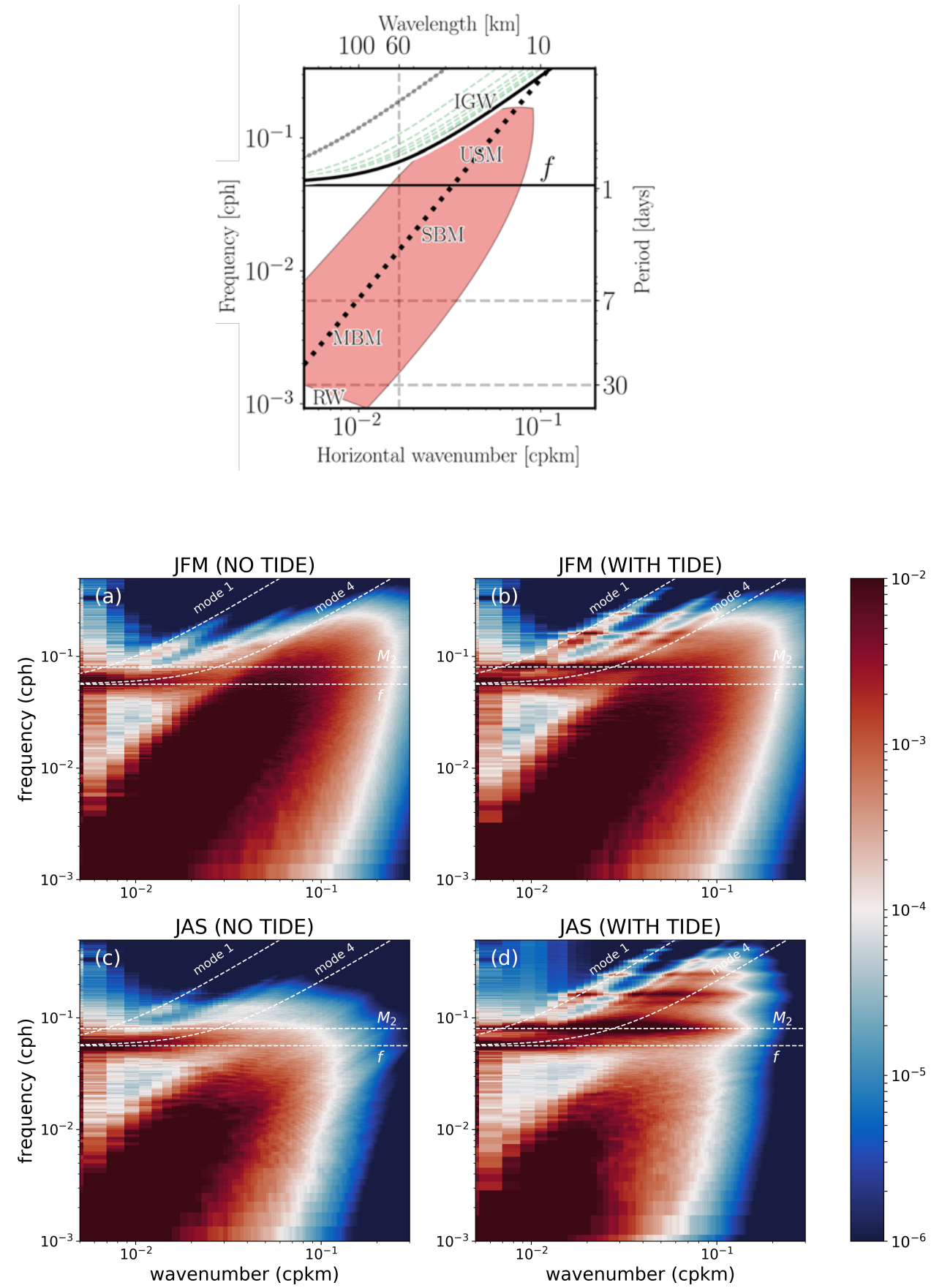

Figure 2. Top panel: A schematic of the observable dynamical regimes with different classes of motions in the ocean (adapted from Torres et al., 2018). These classes of motions starting with low frequency, small wavenumber motions to high frequency frequency, high wavenumber motions are Rossby waves (RW), mesoscale balanced motions (MBM), submesoscale balanced motions (SBM), unbalanced submesoscale motions (USM) and internal gravity waves (IGW). Bottom four plots: Surface KE frequency-wavenumber spectra computed from hourly outputs of eN60-NT (no tides) and eN60-WT (with tides) for winter (JFM) and summer (JAS) time. 

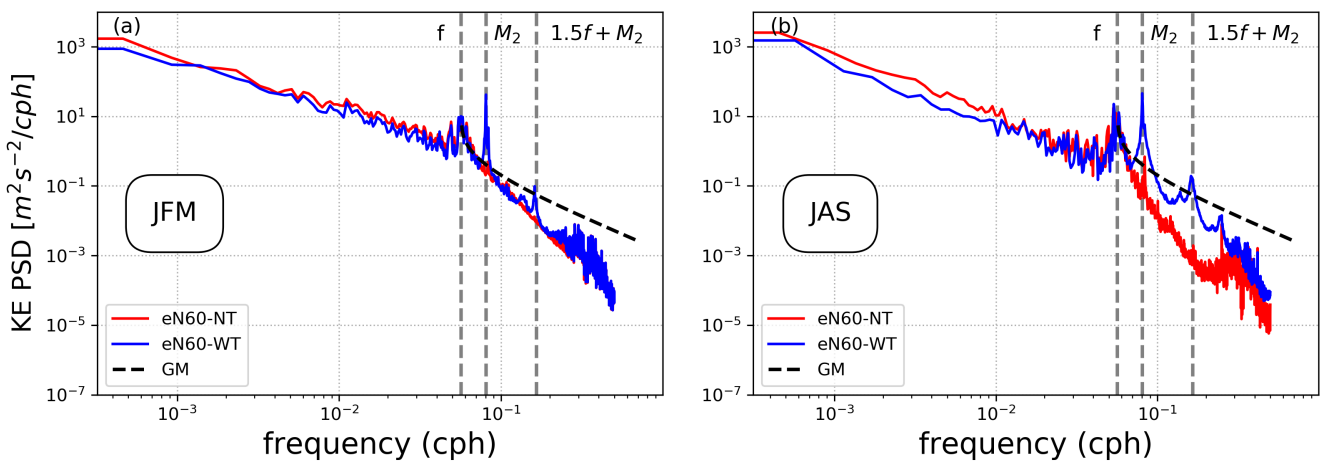

Figure 3. Surface KE frequency spectral density, obtained by integrating the $\omega-K$ spectra over all wavenumbers for eN60-NT (no tides) and eN60-WT (with tides). The three grey dash lines represent the inertia frequency $f$, the $\mathrm{M}_{2}$ tidal frequency, and $1.5 f+\mathrm{M}_{2}$. The dashed black line represents the estimate of the Garrett-Munk spectra computed with reference values of total energy of the internal wavefield and stratification set to $E_{0}=6.3 e^{-5} \mathrm{~m}^{2} \mathrm{~s}^{-2}$ and $N_{0}=5.2 e^{-3} \mathrm{~s}^{-1}$, respectively (Garrett \& Munk, 1975; Cairns \& Williams, 1976; Müller et al., 2015). (a) Winter (JFM) and (b) Summer (JAS). 

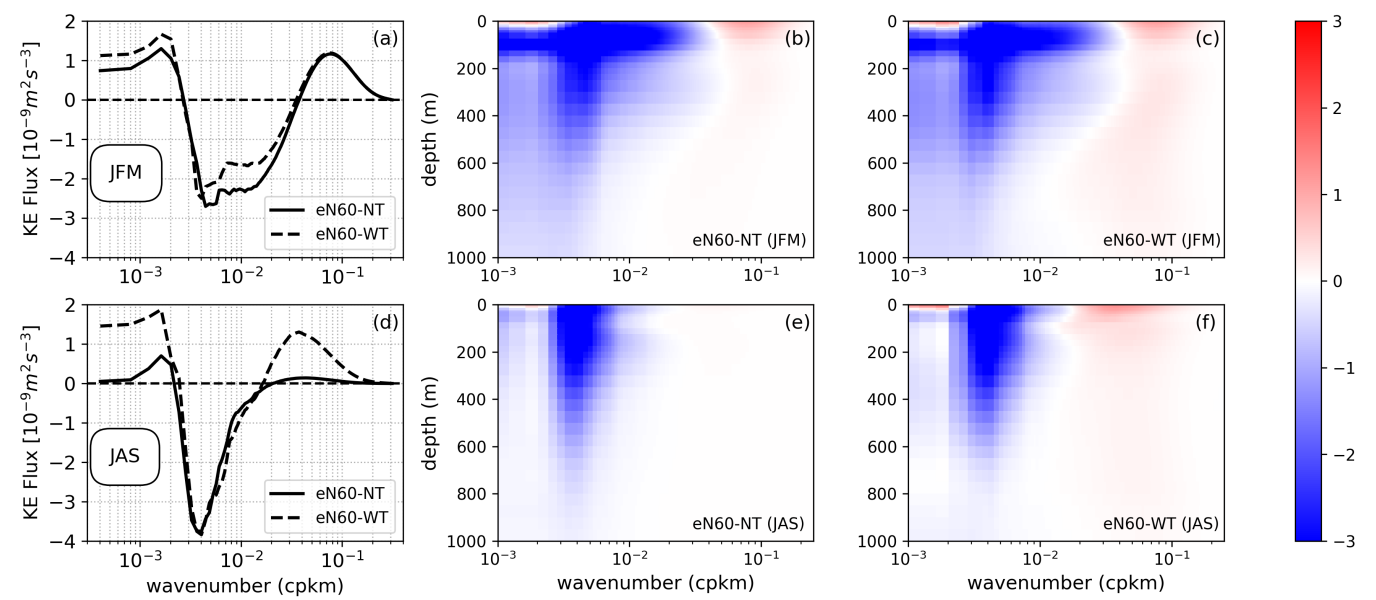

Figure 4. Surface KE spectral flux computed from hourly outputs of eN60-NT (no tides) and eN60-WT (with tides). Summer : July, August and September. Winter : January, February and March. (b,c,e,f) Winter and summer averages of KE spectral flux as a function of depth for eN60-NT and eN60-WT. 

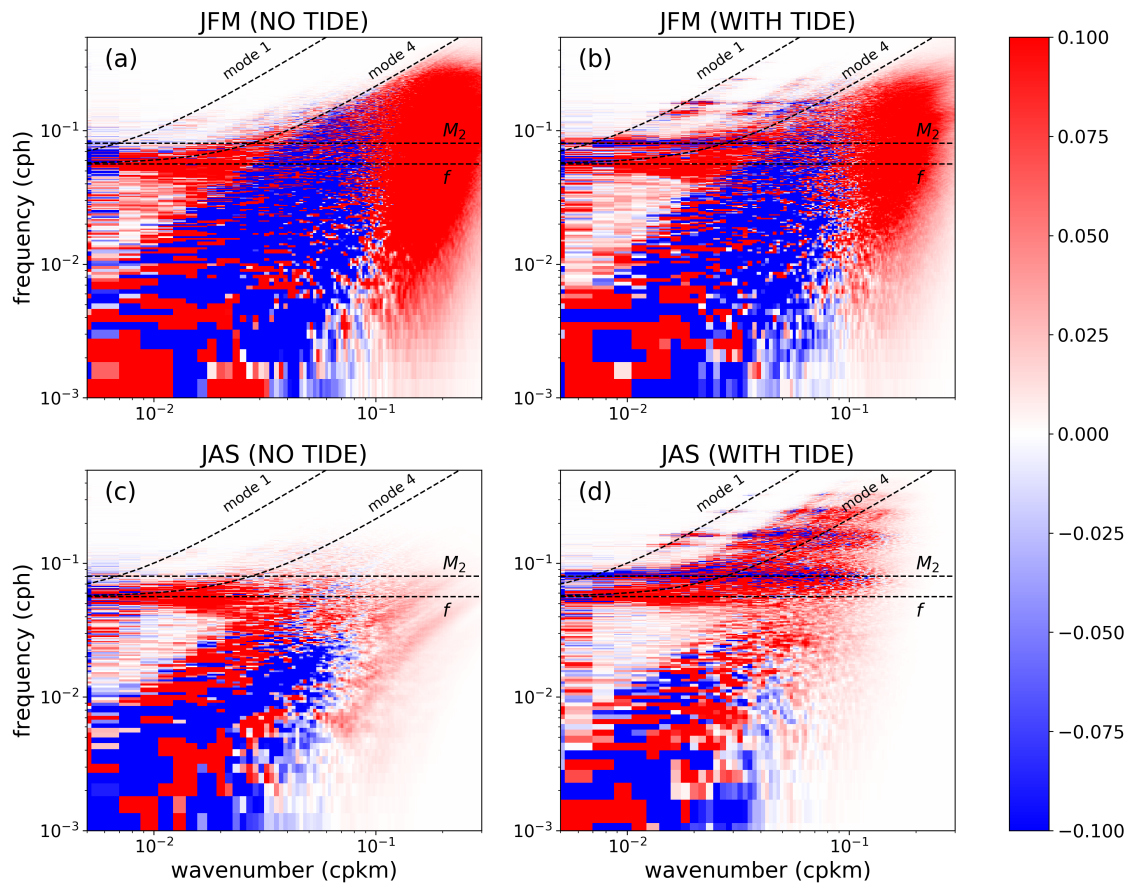

Figure 5. Surface KE transfer in frequency-wavenumber space computed from hourly outputs of eN60-NT (no tides) and eN60-WT (with tides) for winter (JFM) and summer (JAS) time. 